\title{
Per una didattica degli errori. A cento anni dalla nascita di Gianni Rodari
}

\author{
di Vanessa Roghi
}

Ott 23, $2020|\underline{\text { In evidenza, }} \underline{\text { Pensare la didattica }}| \underline{0} \mid$

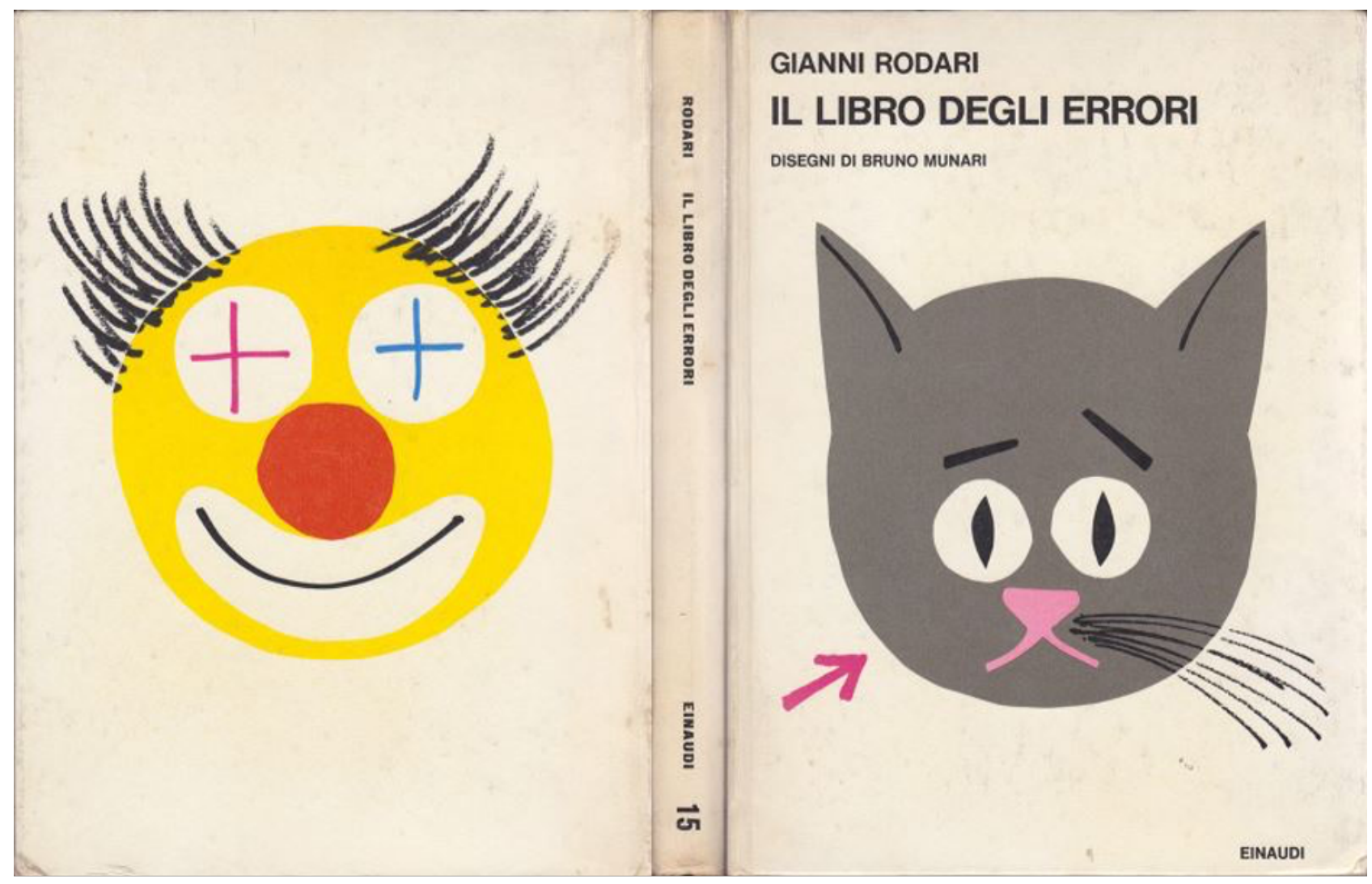

Copertina del volume "Il libro degli errori" di Gianni Rodari

\section{Buon compleanno Gianni Rodari!}

Il 23 ottobre 1920 nasceva a Omegna Gianni Rodari. Un compleanno che oggi, egli stesso, autore di tanta letteratura per l'infanzia e per tutti gli adulti che hanno voglia di comprendere la bellezza del gioco dell'apprendere, avrebbe trovato il modo di celebrare con la leggerezza e la giocosità che lo caratterizzavano. Ma sotto il velo della sua impareggiabile levità, Rodari ha saputo essere molto di più. E molto altro. Un giornalista, un poeta, un partigiano, un pedagogista, un autore di programmi televisivi. Un riferimento ineludibile per la storia della scuola e della pedagogia italiane. Un innovatore. Un inguaribile sognatore. Un osservatore attentissimo del proprio tempo e un instancabile lavoratore per costruire una società migliore, partendo dal mondo dell'infanzia. Quanta storia sociale troviamo, non troppo fra le righe, nella sua vastissima produzione e nelle gaffes tragicomiche del professor Grammaticus. E quanti suggerimenti e riflessioni, ancor oggi, per gli insegnanti di ogni disciplina. Sì, perché la forma e lo spessore delle parole che con tanta passione Rodari ha scrutato, analizzato, spezzato e ricomposto, coltivato e con le quali ha giocato, non servono solo ad apprendere correttamente la lingua italiana. Come prima di lui Don Milani, o Tullio De Mauro e Mario Lodi suoi contemporanei, Rodari ha insegnato che le parole non sono mai neutre. Servono a capire il mondo e ad interpretarlo, a camminare consapevolmente dentro il fluire 
della storia e a rappresentarla. E che ogni tempo ha le proprie parole, le proprie grammatiche e $\mathrm{i}$ propri errori.

\begin{abstract}
Gianni Rodari è stato uno dei più interessanti intellettuali italiani del Novecento. La sua biografia e la sua produzione culturale sono ben più vasti dei libri che tutti conoscono: in questo articolo si fa riferimento alla sua ricerca sugli errori e la loro "didattica" all'interno della definizione di una linguistica democratica che negli anni Sessanta trova importanti interpreti a partire da Tullio De Mauro la cui riflessione molto deve anche a Gianni Rodari.
\end{abstract}

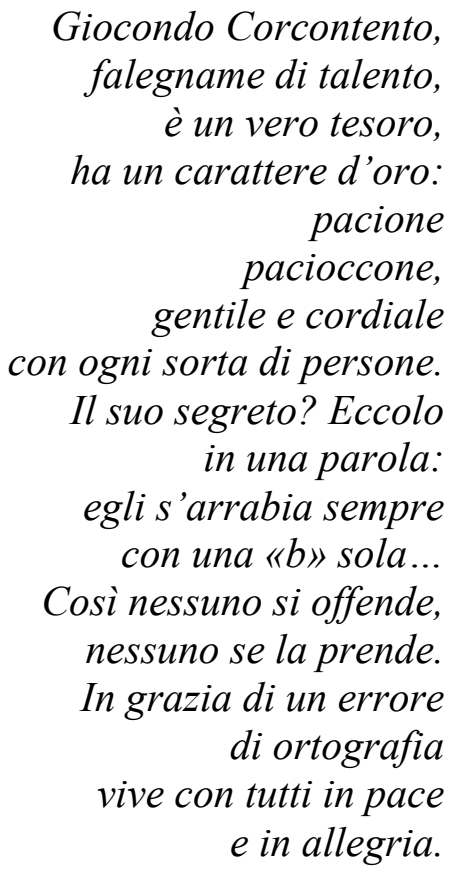

Gianni Rodari

Per molti anni mi sono occupato di errori di ortografia: prima da scolaro, poi da maestro, poi da fabbricante di giocattoli, se mi è permesso di chiamare con questo bel nome le mie precedenti raccolte di filastrocche e di favolette. Talune di quelle filastrocche, per l'appunto dedicate agli accenti sbagliati, ai «quori» malati, alle «zeta» abbandonate, sono state accolte - troppo onore! perfino nelle grammatiche. Questo vuol dire, dopotutto, che l'idea di giocare con gli errori non era del tutto eretica. Vale la pena che un bambino impari piangendo quello che può imparare ridendo? Se si mettessero insieme le lagrime versate nei cinque continenti per colpa dell'ortografia, si otterrebbe una cascata da sfruttare per la produzione dell'energia elettrica. Ma io trovo che sarebbe un'energia troppo costosa. Gli errori sono necessari, utili come il pane e spesso anche belli: per esempio, la torre di Pisa. Questo libro è pieno di errori, e non solo di ortografia. Alcuni sono visibili a occhio nudo, altri sono nascosti come indovinelli. Alcuni sono in versi, altri in prosa. Non tutti sono errori infantili, e questo risponde assolutamente al vero: il mondo sarebbe bellissimo, se ci fossero solo i bambini a sbagliare. Tra noi padri possiamo dircelo. Ma non è male che anche $i$ ragazzi lo sappiano. E per una volta permettete che un libro per ragazzi sia dedicato ai padri di famiglia, e anche alle madri, s'intende, e anche ai maestri di scuola: a quelli insomma che hanno la terribile responsabilità di correggere, senza sbagliare, i più piccoli e innocui errori del nostro pianeta[1]. 


\section{Il libro degli errori}

La prefazione al Libro degli errori Gianni Rodari la scrive fra il 1963 e il 1964: nel suo carteggio con l'editore Einaudi si parla spesso di questa 'paginetta' che dovrebbe racchiudere il senso della sua nuova raccolta di storie e filastrocche. Anche se Il libro degli errori nuovo non lo è del tutto: racchiude, infatti, il lavoro, le riflessioni, le passioni che Gianni Rodari ha messo a punto nel corso degli anni, da quando ha iniziato a frequentare i bambini, nel 1938, come maestro[2].

Ora, nel 1964, Gianni Rodari è diventato uno scrittore famoso, non solo grazie alla collaborazione con Einaudi ma anche a quella con «Il corriere dei piccoli» sul quale scrive dal 1961 grazie a una felice intuizione del suo direttore, Guglielmo Zucconi, che ha voluto questo scrittore comunista come collaboratore del più venduto fra i periodici italiani per l'infanzia.

Scrive a Daniele Ponchiroli, prezioso redattore einaudiano: se nel libro c'è il risvoltino «io credo che in esso - tra l'altro - andrebbe detto che è adattissimo per gli scolari delle elementari, può interessare e divertire anche i ragazzi della media unica (è noto che i professori delle medie si lamentano dell'ortografia delle loro vittime)»[3]. E ancora: il libro dovrebbe essere

una specie di panorama nazionale degli errori tipici: quello milanese di usare la 'esse' per la 'zeta', quello veneto di dimezzare le doppie consonanti, quello sardo di raddoppiare la ' $t$ ' e così via. Questa Italia sbagliata, però, non doveva né poteva diventare un vero e proprio schedario ortografico (ecco, però, uno schedario che sarebbe assai utile alla scuola): doveva restare un libro per bambini. E, se permettete, doveva divertire anche lo scrittore. La mia parte di divertimento consisteva non tanto nel dar la caccia agli errori, quanto nello scoprire il loro risveglio ideologico[4].

Il risveglio ideologico degli errori è quello, per esempio, raccontato in Attenti al tran dove il professor Grammaticus, protagonista dell'avventuroso viaggio attraverso gli errori di ortografia, si accorge della svista di un operaio che su un cartello scrive «Attenti al tran!». Cos'è un tran, si chiede il professore.

«Perché io so che cos'è un tram, con la emme; ma quella roba lì non la conosco». «Be', ci stia attento lo stesso" gli risponde l'operaio.

Il professore era visibilmente indignato. Tanto visibilmente che un passante gli chiese: «Lè è successo qualcosa?» «Non a me - sbottò il professore, - all'ortografia!». Il passante lesse il cartello e sorrise. "Sorride? Beato lei! A me queste cose mi fanno piangere». "Pensavo - disse il passante. - In fondo quel cartello non è sbagliato come crede lei». "Benissimo. Allora ci scriva addirittura: "attenti al trantran"». «Ecco. Ė proprio quello che stavo pensando. Il tram è pericoloso, ma il "TRANTRAN" è più pericoloso ancora. Il tram può spezzare una gamba, ma il "trantran” può uccidere il pensiero. Non è peggio?» Il professor Grammaticus rimase a meditare sulle parole del passante. E se ci penserete un po' anche voi, non vi sarà difficile capirle[5].

Il trantran è un errore blu o rosso? Sicuramente un errore rosso, come spesso lo sono quelli commessi dagli adulti. Ma l'errore peggiore alla fine lo compie proprio il professor Grammaticus in persona in un'altra storia del Libro degli errori dal titolo Essere e avere: il nostro eroe, infatti, viaggiando in treno, ascolta per caso la conversazione dei suoi compagni di scompartimento. Sono operai meridionali, emigrati all'estero in cerca di lavoro: tornati in Italia per le elezioni, stanno ripartendo. «Io ho andato in Germania nel 1958», diceva uno di loro. «Io ho andato prima in Belgio, nelle miniere di carbone. Ma era una vita troppo dura». Il professor Grammaticus li ascolta in 
silenzio, trema per l'indignazione: non può tollerare un simile scempio delle regole grammaticali. Alla fine, sbotta:

«Ho andato! Ho andato! Ecco di nuovo il benedetto vizio di tanti italiani del Sud di usare il verbo avere al posto del verbo essere. Non vi hanno insegnato a scuola che si dice: "sono andato"»? Gli emigranti tacquero, pieni di rispetto per quel signore tanto perbene, con i capelli bianchi che gli uscivano di sotto il cappello nero. "Il verbo andare», continuò il professor Grammaticus, "è un verbo intransitivo, e come tale vuole l'ausiliare essere». Gli emigranti sospirarono. Poi uno di loro tossi per farsi coraggio e disse: "Sarà come lei dice, signore. Lei deve aver studiato molto. Io ho fatto la seconda elementare, ma già allora dovevo guardare più alle pecore che ai libri. Il verbo andare sarà anche quella cosa che dice lei». "Un verbo intransitivo». "Ecco, sarà un verbo intransitivo, una cosa importantissima, non discuto. Ma a me sembra un verbo triste, molto triste. Andare a cercar lavoro in casa d'altri... Lasciare la famiglia, i bambini». Il professor Grammaticus cominciò a balbettare. "Certo... Veramente... Insomma, però... Comunque si dice sono andato, non ho andato. Ci vuole il verbo "essere": io sono, tu sei, egli è...». "Eh, disse l'emigrante, sorridendo con gentilezza, io sono, noi siamo!... Lo sa dove siamo noi, con tutto il verbo essere e con tutto il cuore? Siamo sempre al paese, anche se abbiamo andato in Germania e in Francia. Siamo sempre là, è là che vorremmo restare, e avere belle fabbriche per lavorare, $e$ belle case per abitare». E guardava il professor Grammaticus con i suoi occhi buoni e puliti. E il professor Grammaticus aveva una gran voglia di darsi dei pugni in testa. E intanto borbottava tra sé: «Stupido! Stupido che non sono altro. Vado a cercare gli errori nei verbi... Ma gli errori più grossi sono nelle cose!»[6].

Nata negli anni Cinquanta, la riflessione sugli errori diventa il cardine della ricerca rodariana sulla lingua: se prendiamo infatti i primi interventi, quelli pubblicati sull'«Unità» o sul «Pioniere» vediamo chiaramente come lo scrittore di Omegna sostituisca a poco a poco all'intento pedagogico di stampo tradizionale, mutuato dalla pedagogia sovietica, un intento sovvertitore dello stato di cose reale, un progetto che dalla grammatica passa alla rivoluzione senza soluzione di continuità[7].

La riflessione sulla lingua e le sue variabili (ancorché inesatte), insomma, non è fine a sé stessa, a un uso scolastico, è parte di un processo che mira da un lato ad impossessarsi delle parole con tutti i loro usi, dall'altra a stravolgere il senso dell'apprendimento che non può, non deve essere finalizzato al risultato, al voto, alla promozione, ma al processo, alla collaborazione, alla crescita personale e collettiva del gruppo classe attraverso, appunto, il gioco, a partire dall'errore[8].

\section{Genealogia dell'errore}

Gianni Rodari queste cose le impara anche dai maestri del Movimento di cooperazione educativa: senza di loro, infatti, il suo sguardo resterebbe molto più tradizionalmente legato all'ideologia dell'impegno propugnata da Antonio Gramsci per esempio; molto letto inoltre nei primi anni Cinquanta è Poema pedagogico di Anton Semënovič Makarenko che viene pubblicato Italia nel 1952 con la prefazione di Lucio Lombardo Radice. Gramsci e Makarenko rimangono due punti di riferimento ineludibili, dal Poema Rodari ricava l'importanza del lavoro collettivo: Makarenko «ha creato il collettivo come unità minima, non soltanto pedagogica, ma anche educativa ed esistenziale nel senso più alto e onnicomprensivo del concetto». «La vita è co-esistenza, compassione, collaborazione, ogni tanto conflitto. Tale prefisso "co, con" rende forse al meglio l'essenza stessa del Poema, ed echeggia l'ideologia gork'iana che sottende tutta l'opera e che possiamo condensare nella massima "un uomo solo, per quanto grande, è pur sempre solo"»[9]. 
Alle letture comuniste, nel bagaglio culturale rodariano, si aggiungono gli esiti di quella meravigliosa rivoluzione innescata dall'attività editoriale di case editrici come Einaudi, che, come ha scritto Luisa Mangoni, "pensa libri” per un'Italia nuova e democratica:

come tanti altri, ho scoperto l'etnografia e l'etnologia quando me l'ha fatta scoprire Pavese, inventando per Einaudi una famosa collana. Ho scoperto la linguistica un bel po' di anni dopo aver abbandonato l'università, dove riuscii, sicuramente con l'aiuto della medesima, a non averne il minimo sospetto. Almeno una cosa ho imparato: che quando si ha a che fare con $i$ bambini, e si vuol capire quel che fanno e quel che dicono, la pedagogia non basta e la psicologia non arriva a dare una rappresentazione totale delle loro manifestazioni. Bisogna studiare altre cose, appropriarsi di altri strumenti di analisi e di misura. Anche farlo da autodidatti non guasta niente. Anzi[10].

Da autodidatta, dunque, Rodari si avvicina alla semiotica, all'insiemistica, alle neuroscienze (anche se ancora non si chiamano così). Da ogni ricerca che incontra, in ogni studioso che l'appassiona, isola questioni che lo interessano come quella del gioco, che può manifestarsi sotto forma di domanda, enigma o di indovinello. Il piacere di «quella forma quanto mai primitiva di trionfo sull'ignoto che consiste nello scoprire ciò che ci è familiare, nascosto sotto sembianze sconosciute»[11]. Ogni forma di capovolgimento della realtà può essere utile per immaginare, esercitare il pensiero utopico: anche l'errore.

\section{La riforma delle scuole medie}

Il libro degli errori esce nel 1964, un anno dopo l'entrata in vigore della riforma delle scuole medie. Un anno dopo la pubblicazione di C'è speranza se questo accade al Vho di Mario Lodi e della Storia linguistica dell'Italia unita di Tullio De Mauro che in copertina riporterà la nota citazione gramsciana:

Ogni volta che affiora, in un modo o nell'altro, la quistione della lingua, significa che si sta imponendo una serie di altri problemi: la formazione e l'allargamento della classe dirigente, la necessità di stabilire rapporti più intimi e sicuri tra i gruppi dirigenti e la massa popolarenazionale, cioè di riorganizzare l'egemonia culturale[12].

Il lavoro rodariano si inserisce all'interno di questa riflessione comune, entra a pieno titolo a far parte di quel tessuto democratico, come l'ha definito De Mauro, che si pone il problema di dare una risposta alla questione della cittadinanza degli italiani che non possiedono la lingua (lessico mutuato dalla Lettera a una professoressa che uscendo nel 1967 tira le somme di buona parte di questa riflessione), che si pone il problema di riorganizzare l'egemonia culturale.

Lo sguardo rodariano, tuttavia, non entra affatto in modo strutturale nella scuola italiana degli anni Sessanta, la scuola alla quale lo scrittore si rivolge. Nemmeno gli insegnanti coinvolti nella riforma delle scuole medie del 1963 e nella conseguente riflessione sull'insegnamento dell'italiano accolgono, se non in modo individuale e del tutto casuale, dunque, il Libro degli errori come uno strumento didattico, questo lo racconta Tullio De Mauro che nel 1969 scrive un saggio "a proposito degli sbagli di ortografia":

chi ha preso parte a corsi di aggiornamento lo sa bene: per i colleghi che insegnano nelle scuole elementari e medie i problemi della didattica della lingua più angosciosi e drammatici sono $i$ problemi dell'ortografia. Si parla di Saussure o di Prieto o di Chomsky, si parla di sintassi 
aristotelica e di sintassi moderna, si parla di livelli linguistici, di interferenze tra dialetto e lingua; ma poi quando si è al dunque della discussione, viene sempre il momento in cui si alzano in due, tre, dieci insegnanti e cominciano a parlare dei guai dell'ortografia: "livelli sintattici? Magari ma $i$ miei alunni non sanno nemmeno come scrivere correttamente le parole. "Valore colloquiale e letterario delle espressioni? Ma se mi scrivono egli a senza h e terribbile con due b!" "Nuove tecniche didattiche per l'arricchimento del vocabolario? Benvenute! Ma diteci prima cosa dobbiamo fare con i ragazzi che scrivono conosciente con la i e deficiente senza i[13].

Gli stessi compagni del PCI che interpretano le posizioni ufficiali e scrivono sui periodici di partito, secondo Rodari che se ne lamenta con l'amico Paolo Spriano, sono tiepidi nei confronti di una proposta che appare troppo ludica, sovvertitrice di regole che alla fine condividono tutti senza capire bene perché.

Come ha scritto Silvia Vinante «quanto sono cambiate le grammatiche scolastiche in un secolo di scolarità obbligatoria? Poco se andiamo a confrontarle. Ciò che in un secolo e mezzo di studi linguistici è stato studiato nei dipartimenti, sembra non entrare nelle grammatiche scolastiche». Se non in minima parte nella seconda metà degli anni Settanta, in seguito alle Dieci tesi del Giscel, «la teoria che sta alla base, la concezione di grammatica, è molto simile a quella che vediamo in una grammatica degli anni '30. Prima si fa l'articolo, poi si fa il nome, per fare un esempio».

Eppure Gianni Rodari partecipa a un movimento che negli stessi anni si pone il problema del rinnovamento della didattica a tutto tondo: Maria Corda Costa e Tullio De Mauro fra il 1963 e il 1966 tengono seminari comuni di filosofia del linguaggio e pedagogia alla Sapienza nella Facoltà di Lettere di Roma; e poi le iniziative di quelli che allora si chiamavano "comuni democratici": realtà amministrative che incidono profondamente sulla formazione del corpo insegnante (pensiamo al comune di Reggio Emilia).

Scrive De Mauro:

vale la pena ricordare che, ancora a metà degli anni Sessanta, erano ostracizzati in Italia tutti gli indirizzi di ricerca linguistica che non avessero carattere filologizzante, perciò poco spazio avevano gli studi di linguistica descrittiva e funzionale, meno ancora, se possibile, gli studi di teoria del linguaggio e delle lingue, e nessuno infine, avevano le ricerche di tipo applicato, fatte per migliorare la pratica dell'educazione linguistica[14].

Eppure, la "quistione della lingua" è tema attuale e dibattuto, persino sui quotidiani nazionali: nota la polemica innescata da Pier Paolo Pasolini nel 1964, una polemica che scuote gli ambiti specialistici ma che troppe poche ricadute ha nell'insegnamento scolastico[15].

\section{Il tessuto democratico}

Secondo Tullio De Mauro le svolte rilevanti sono facilmente ricostruibili: nel 1966 un dibattito avviato sulla rivista «Il contemporaneo» sull'insegnamento dell'italiano; la nascita della SLI, società linguistica italiana nel 1967 e la sua affermazione come «tollerante casa di tutti» fino ai primi anni Settanta quando Vygotskij, Gramsci, Saussure vengono riletti alla luce l'uno dell'altro, come fa, per esempio Gianni Rodari quando scrive La grammatica della fantasia nel 1973.

Ma ho corso troppo: faccio un passo indietro per tornare a quel 1964 quando esce il Libro degli errori e Rodari inizia a osservare la scuola nella sua realtà, da vicino. Sua figlia Paola, infatti, nata 
nel 1957, è diventata una piccola scolara e quello che il genitore Rodari tocca con mano è ben diverso da quello che ha sperato di trovare e di contribuire a cambiare nel corso degli anni. La scuola è, malgrado tutti i discorsi e gli sforzi di pochi insegnanti illuminati, il regno dove governa, anzi comanda, re voto che l'errore lo punisce. Certo non lo premia, mai, nemmeno per trasformarlo in spunto di riflessione. Tutto si fa per la valutazione, in vista di quella. Una scuola senza voto «è triste, per certi insegnanti, come il brodo senza tortellini»[16].

Così sul «Corriere dei Piccoli» Rodari, il 22 ottobre 1967, pubblica una giocosa intervista al voto:

Domanda. Buongiorno, signor Voto. Avendo appreso che è tornato a scuola anche lei, abbiamo pensato di rivolgerle alcune domande. Lei si ritiene veramente importante nella vita di un ragazzo? Risposta. - Ecco, vede: se considero che dipendono da me promozioni e bocciature, premi, castighi, liti in famiglia e, qualche volta, perfino fughe nelle isole Molucche, mi sento molto, molto importante. Però... D. - Però? R. - Però non dimentico che il mondo è pieno di cose più importanti di me. Per esempio, quel che s'impara è più importante del voto che si riceve. Ci sono cose più belle di un dieci, per esempio una gita in campagna; e cose più brutte di uno zero, per esempio la fame in India. D. - Non le sembra di buttarsi un po' giù? R. - È che non voglio darmi delle arie. So che incremento il consumo delle matite rosse e blu, che molti insegnanti mi tengono segreto come un messaggio dell'agente 007, che il mio prestigio nei registri è altissimo, ma sono anche il primo a sapere che è difficile misurare un ragazzo in cifre. I voti non sono pesi da farmacista che spaccano il milligrammo: sono approssimazioni. D. - Allora, secondo lei, un brutto voto non sarebbe un voto brutto? R.- Non mi fraintenda. Dico che Carletto può essere un ottimo ragazzo anche se gli capita di prendere un quattro in italiano. Marconi fu bocciato due volte alla licenza tecnica, non poté nemmeno iscriversi all'università, e poi inventò la radio, lasciando i suoi brutti voti di princisbecco: lo so di sicuro perché uno dei suoi quattro era un po' mio parente. Giovanni Pascoli, agli esami di ginnasio, prese quattro in matematica, e altri pessimi voti in altre materie scientifiche: oggi è materia di studio anche lui. Non parliamo del solito Verdi, che fu bocciato al conservatorio di musica. Più di un genio, a scuola, prendeva brutti voti: vuol dire che non si era ancora rivelato, ecco tutto. C'è chi matura prima e chi dopo. Un brutto voto è un nemico che si può battere, non è un'eruzione del Vesuvio, di fronte alla quale non rimane che scappare. D. - Esiste una scienza per dare sempre voti giusti, senza sbagliare? R. - Esiste, e ha un nome difficilissimo: si chiama "docimologia". Ben pochi la conoscono, e del resto non è una scienza indispensabile. Esistono invece scuole dove $i$ voti se li danno maestri e ragazzi insieme, discutendone le ragioni. Esistono scuole dove i voti sono aboliti[17].

Così parallelamente sul «Giornale dei genitori», di cui diventa direttore nel 1968, e sul «Corriere dei piccoli» $\mathrm{o}$ «Paese sera», Rodari conduce la sua battaglia tutta politica per una didattica creativa dell'errore, contro il voto usato come arma, a favore dell'emancipazione del bambino da scolaro a cittadino.

Nel 1973 su «La via migliore», altro periodico a cui collabora, scrive:

Ecco un ragazzo refrattario alla corretta ortografia. Come si comportava con lui la scuola del voto? Seminava il suo scritto di grossi segni rossi e blu, in fondo alla pagina scriveva il numero quattro, il maestro aveva compiuto il suo dovere, il ragazzo aveva il fatto suo. Come si comporta la scuola senza voti? Constata che quel ragazzo sbaglia le acca e le doppie, scambia apostrofi e accenti, scrive "quore" con la " $q$ ". Dunque ha bisogno di particolari esercizi che lo aiutino ad assorbire i modelli corretti. Gli si mette a disposizione uno schedario ortografico con esercizi graduati. Lo si aiuta a trovare il suo esercizio e si controlla che non lo trascuri. Intanto la scuola si chiede: come mai questo ragazzo non sente ancora il bisogno di scrivere correttamente per poter comunicare con gli altri? e se invece di fargli fare degli esercizi gli facessimo scrivere delle lettere 
ai suoi compagni, non gli verrebbe più naturale sforzarsi di non fare errori? Il voto non risponde a nessuna di queste domande. L'alternativa al voto (...) è un altro modo di fare scuola. É una scuola più seria, nella quale si cerca la giusta maniera di insegnare a tutti a scrivere, leggere, calcolare, capire, ragionare, eccetera[18].

\section{Il trantran}

L'errore, il voto, due aspetti del trantran scolastico immutabili, parti costitutive di quella lunga durata che caratterizza la scuola italiana fin dagli anni dell'Unità sui quali Gianni Rodari riflette tutta la vita. "Le cose sono andate sempre così", allora. Il trantran scolastico è immutabile? No, dice Rodari, le cose sono andate così perché qualcuno, noi o altre e altri prima di noi, ce le ha fatte andare così. E se sono state fatte andare così, potremmo anche provare a farle andare in altro modo. La prima cosa da fare è prendere possesso della lingua attraverso le parole: giocando, sbagliando, ripetendo magici e antichi rituali come quello del ripetere la parola all'infinito. "Giocare con le parole ci serve a non subire il mondo così com'è, a immaginarlo diverso. Questo da una parte ci prepara a intervenire nelle cose per cambiarle, se vorremo, dall'altra parte ci prepara a capire meglio le cose (e le parole) così come sono. Ma capire per prepararci a trasformarle». Così facendo, scrive Tullio De Mauro:

vedremo fra le parole e i giochi balenare il serio lavoro di esercizio delle capacità di pensare e capire gli altri, di essere solidali con gli altri, di essere liberi per gli altri. (Non fidatevi mai, non fidatevi mai di chi dice che bisogna essere liberi e basta. Chiedetegli sempre: liberi si, ma da che? Liberi si, ma per che?). Ancora una volta, come sempre, riflettere sulle parole, giocare con le parole, ci riporta a chi usa le parole, alle cose che facciamo e capiamo usando le parole (e non c'è cosa che gli esseri umani riescano a fare che non sia intrisa, intessuta di atti di comprensione e produzione di frasi e di parole) [19].

L'imperatore,
l'impiegato,
l'impiastratore,
l'impiccato
vivono nella stessa pagina
e non si dicono mai:
Buongiorno, come stai... $[20]$

\section{BIBLIOGRAFIA}

- T. De Mauro, Scuola e linguaggio, Editori Riuniti, Roma 1977.

- M. Lodi, C'è speranza se questo accade al Vho, Edizioni Avanti, Milano 1963.

- G. Rodari, Caro Ponchiroli, 17 febbraio 1964, in Lettere a don Julio Einaudi, Hidalgo Editorial e ad altri queridos amigos, 1952-1980, a cura di S. Bartezzaghi, Einaudi, Torino 2005.

- G. Rodari, Come è nato il libro degli errori, in «Noi donne», 45, 14 novembre, 1964, p. 108.

- G. Rodari, Intendersi bene sulla scuola seria, in «Paese sera», 12 febbraio 1978, p. 3.

- G. Rodari, Il libro degli errori, Einaudi, Torino 1964.

- G. Rodari, La grammatica della fantasia, Einaudi, Torino 1973.

- G. Rodari, La scuola senza voti, in «Paese sera», 25 settembre 1977, p. 3. 
- V. Roghi, La lettera sovversiva. Da don Milani a De Mauro il potere delle parole, Laterza, Roma Bari 2017

- V. Roghi, Lezioni di Fantastica. Storia di Gianni Rodari, Laterza, Roma-Bari 2020

Note:

[1] G. Rodari, Tra noi padri, in Il libro degli errori, Einaudi, Torino 1964, p. 9.

[2] Mi permetto di rinviare al mio Lezioni di Fantastica. Storia di Gianni Rodari, Laterza, RomaBari 2020.

[3] G. Rodari, Caro Ponchiroli, 17 febbraio 1964, in Id., Lettere a don Julio Einaudi, Hidalgo

Editorial e ad altri queridos amigos, 1952-1980 a cura di S. Bartezzaghi, Einaudi, Torino 2005, p. 40 .

[4] G. Rodari, Come è nato il libro degli errori, in «Noi donne», 45, 14 novembre, 1964, p. 108.

[5] G. Rodari, Il trantran, in Il libro degli errori, cit., p. 80.

[6] G. Rodari, Essere e avere, in Il libro degli errori, cit., p. 16.

[7] Sul «Pioniere», esce L'ago di Garda il 22 marzo 1959 «Se, per una volta, mi intrometto nei vostri "affari scolastici" senza essere autorizzato; se mi permetto di consigliarvi lo studio dell'ortografia, non è per fare il ficcanaso, o il pignolo, ma per evitarvi di figurare nel "Museo degli errori", che sto preparando da tempo. In questo Museo vado raccogliendo, con santa pazienza, tutte le bizzarrie che possono nascere da un errore di ortografia. Vi farò degli esempi. Ecco la città di Comò. Non cercatela sull'atlantino della Lombardia: lì dentro trovereste solo la città di Como, senza accento. La città di Comò è frutto di un accento sbagliato. I comaschi di Como (senza accento) passeggiano tranquilli in riva al lago; i comaschi di Comò (la città del mio museo) vivono chiusi nei cassetti, lanciando maledizioni a chi ve li ha imprigionati. Ecco "l'ago di Garda". È bastato un apostrofo sbagliato, e il celebre lago si è trasformato in un sottilissimo filo d'acciaio. La luna cerca vanamente di specchiarsi nelle sue onde: non fa che pungersi il naso. I battelli si sono ritrovati improvvisamente all'asciutto: decisamente "l'ago di Garda" non è navigabile; inutile tentare di attraversarlo a nuoto: può attraversarlo, passando per la cruna, solo un filo di refe. I pesci, tutti morti naturalmente. I danni per l'agricoltura rivierasca, gravissimi. Tutto per un apostrofo fuori posto. Procedendo nella visita al "Museo degli errori" incontriamo uno zio infuriatissimo. Suo nipote ha scritto, nel quaderno d'italiano, che "lo zio è il padre dei vizi". Lo zio protesta: "Nipote degenere! Forse ti ho insegnato a fumare? Forse ti dò di nascosto dalla mamma bottigliette di aranciata da tenere nella cartella per straviziare nelle ore di ricreazione? Forse ti tengo alzato la notte per vedere la televisione?". Tra gli altri esemplari interessanti, posseggo un'intera raccolta di "zeta" abbandonate. Tristissime "zeta", orfanelle mie di dove venite? Una doveva entrare nella parola "risoluzione", ma ne è stata cacciata, perché al suo posto è entrata una "esse": "risolusione". Un'altra, un tipo molto attivo, doveva far parte della parola "azione" e partire, appunto, per un'audacissima impresa: è stata lasciata a terra all'ultimo minuto, e al suo posto si è imbarcata la solita "esse". Il risultato, una "asione" che sarà stata certo un fallimento. Che "azione" bella, coraggiosa, ci può essere senza la zeta? Qui ho nientemeno che un pellerossa. Un "apache". Non sa perché è capitato nel mio museo, rimpiange le libere praterie, i bisonti, i cavalli. Uno scolaro 
doveva scrivere "la pace", ed ha scritto "l'apache": ecco svelato il mistero dell'indiano solitario e senza patria. Scrivere bene le parole è un ottimo allenamento per fare bene le cose. Scriverle male è una maniera di mandarle a gambe all'aria», G. Rodari, L'ago di Garda, «Il Pioniere», 22 marzo 1959, p. 3.

[8] Cfr. per un inquadramento generale F. Cambi, Rodari e l'infanzia, «Scuola e città», 1980, p. 11. F. Cambi, L'immagine "formale” della pedagogia nell'opera di Rodari, in Carmine De Luca (a cura di), Se la fantasia cavalca con la ragione. prolungamenti degli itinerari suggeriti dall'opera di Gianni Rodari: convegno nel decennale della "Grammatica della fantasia”, Reggio Emilia, 10-12 novembre 1982, Juvenilia, Bergamo 1983, pp. 45-52. Per alcune valutazioni della pedagogia rodariana cfr. M. Dallari, Rodari pedagogista e poeta, in «Riforma della scuola», 1980, p. 9; M. Di Rienzo, Opinion-maker per la scuola, in AA. VV., Il favoloso Gianni: Rodari nella scuola e nella cultura italiana, Nuova Guaraldi, Firenze 1982, pp. 93-115.

[9] T. F. Korableva, E. Mettini, Sul Poema pedagogico di A. S. Makarenko, in A. S. Makarenko, Poema pedagogico, a cura di Nicola Siciliani de Cumis, Roma, Albatros, 2009, p. XI. Vedere anche G. Consoli, Romanzo e rivoluzione. Il Poema pedagogico di A. S. Makarenko come nuovo paradigma del racconto, ETS, Pisa 2007.

[10] G. Rodari, La parola che gioca, in Id, La grammatica della fantasia, Einaudi, Torino 1973, p. 179.

[11] G. Rodari, L 'indovinello come forma del conoscere, in ivi, p. 183.

[12] A. Gramsci, Quaderni del carcere, Quaderno 29, § 3.

[13] T. De Mauro, Scuola e linguaggio, Editori Riuniti, Roma 1977, p. 55.

[14] De Mauro, 1977, pp. 7-8.

[15] Per la ricostruzione del dibattito cfr. V. Roghi, La lettera sovversiva. Da don Milani a De Mauro il potere delle parole, Laterza, Roma Bari 2017, pp. 112 sgg.

[16] G. Rodari, Intendersi bene sulla scuola seria, in «Paese sera», 12 febbraio 1978, p. 3.

[17] G. Rodari, La scuola senza voti, in «Paese sera», 25 settembre 1977, p. 3.

[18] G. Rodari, «La via migliore», febbraio 1973 n. 6.

[19] T. De Mauro, Presentazione a Parole per giocare, Biblioteca di Lavoro di Mario Lodi, Luciano Manzuoli, Firenze 1979, p. 3.

[20] T. De Maurio, Coabitazione in Parole per giocare, Biblioteca di Lavoro di Mario Lodi, Luciano Manzuoli, Firenze 1979, p. 12. 